\title{
Pneumobilia in chest radiograph
}

\author{
Li-Hsiu Chen · Hung-Jen Chen • Wei-Chih Liao
}

Received: 5 August 2011 / Accepted: 15 October 2011/Published online: 29 October 2011 (C) SIMI 2011

\section{Case report}

A 64-year-old woman with a history of cholelithiasis and pneumobilia presented to our emergent department (ED) because of a 3-day history of abdominal pain. The pain was dull and in the right upper quadrant (RUQ) without migration or radiation. She also complained of fever, nausea, and vomiting.

She had a history of community-acquired pneumonia 18 months previously, and pneumobilia was found incidentally on chest radiography. The chest radiography at that time revealed pneumonia in the right lower lobe and air in the RUQ of the abdomen (Fig. 1). Abdominal computed tomography (CT) showed air in the biliary tree and intrahepatic duct (IHD) stones, which confirmed that the pneumobilia was due to IHD stones (Fig. 2). Because pneumobilia was asymptomatic, the surgeon suggested no surgical intervention was necessary. After treatment for pneumonia, she was discharged without intervention for the pneumobilia.

In the ED, she appeared acutely ill with no alterations of consciousness. Physical examination revealed a body temperature $38^{\circ} \mathrm{C}$, heart rate 110 beats/min, respiratory rate 30 breaths $/ \mathrm{min}$, and blood pressure $80 / 60 \mathrm{mmHg}$. The bowel sounds were hypoactive, and the abdomen was tender over the RUQ without guarding or rebound tenderness. Laboratory studies revealed a white blood cell

L.-H. Chen · H.-J. Chen · W.-C. Liao ( $\square)$

Department of Internal Medicine, China Medical University Hospital, No. 2, Yuh-Der Road, Taichung City 40447, Taiwan e-mail: weichih.liao@gmail.com

W.-C. Liao

China Medical University, Taichung, Taiwan count of $8.78 \times 10^{9}$ cells/L (87.5\% neutrophils); hemoglobin, $14.0 \mathrm{~g} / \mathrm{dL}$; platelet count, $130 \times 10^{9} / \mathrm{L}$; C-reactive protein, $26.45 \mathrm{mg} / \mathrm{dL}$; aspartate aminotransferase, $108 \mathrm{IU} /$ L; alanine aminotransferase (ALT), 178 IU/L; total bilirubin, $3.62 \mathrm{mg} / \mathrm{dL}$; blood urea nitrogen, $55 \mathrm{mg} / \mathrm{dL}$; and creatinine, $3.4 \mathrm{mg} / \mathrm{dL}$. Abdominal computed tomography (CT scan) demonstrated cholelithiasis, IHD stones, and common bile duct (CBD) dilatation. Under the impression of CBD stones with acute cholangitis and septic shock, emergent choledocholithotomy with T-tube insertion and stone removal were performed. Postoperatively, she was treated with broad-spectrum antibiotics, and was discharged uneventfully on postoperative day 10 .

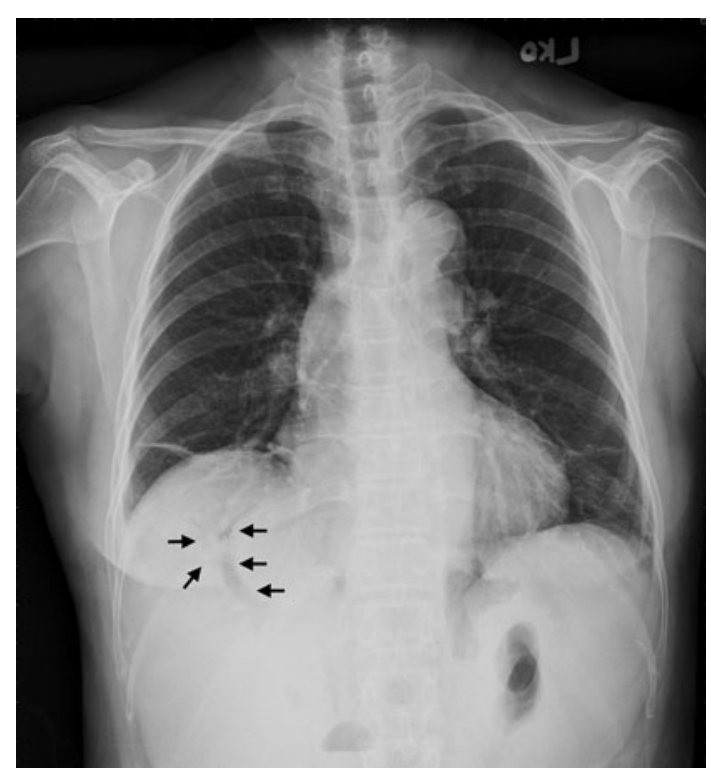

Fig. 1 Chest radiography showed right lower lobe pneumonia and air with a tree-like distribution in the right upper quadrant (arrows) 

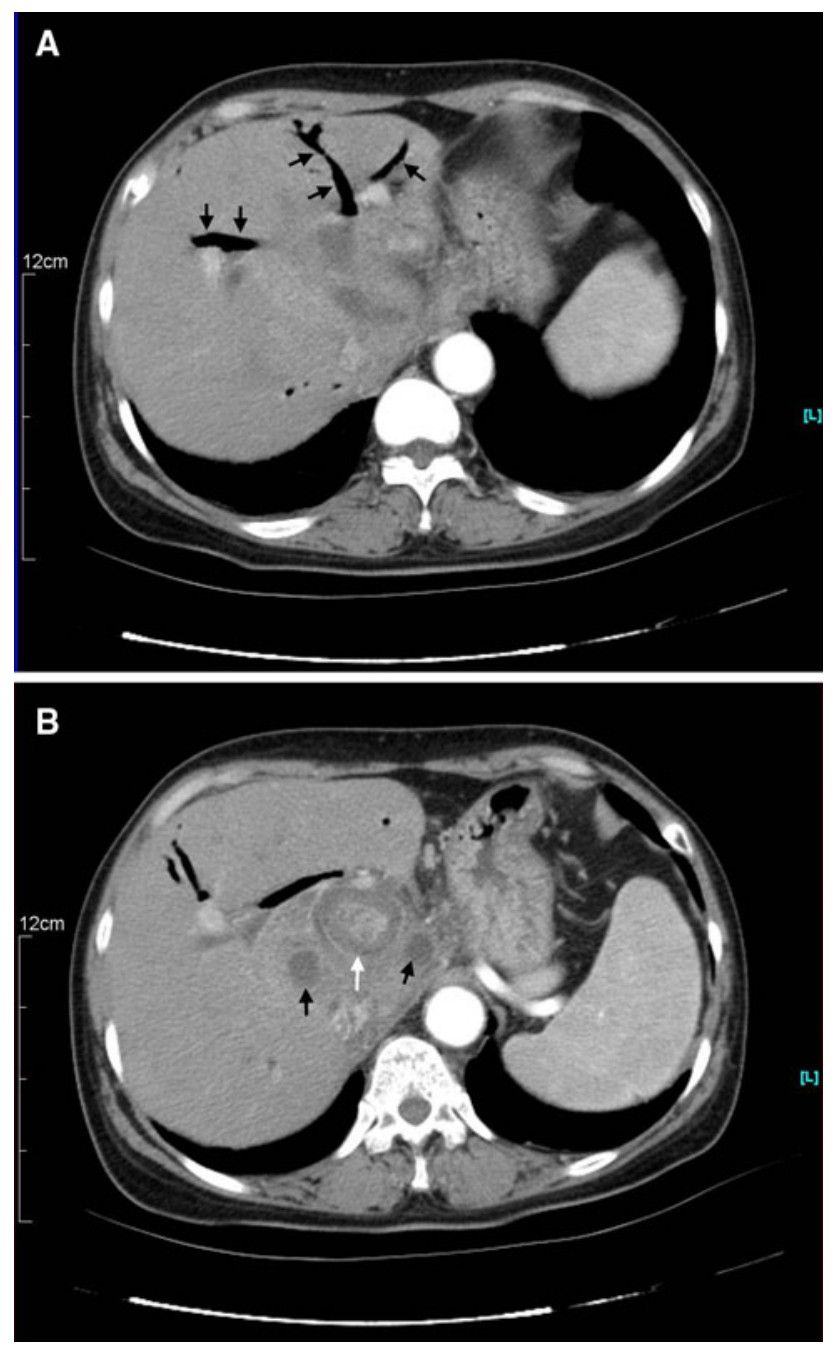

Fig. 2 Abdominal computed tomography. Air in the biliary tract (arrows), dilated intrahepatic ducts (black arrows) and intrahepatic duct stones (white arrow)

Pneumobilia, or the gas in the biliary tract, is suggestive of an abnormal communication between the intestines and the biliary ducts, or an infection by gas-producing organisms. It is usually a relatively benign condition, such as when it is recognized postoperatively; however, it can be a life-threatening condition, such as when due to an emphysematous infectious process [1]. Pneumobilia is more readily recognized on $\mathrm{CT}$ images than magnetic resonance imaging [2]. With careful attention, it can be seen on plain abdominal radiography or even on chest radiography. Prompt diagnosis and early surgical intervention are needed to minimize the significant morbidity and mortality associated with causes requiring surgical management [3]. While we can see delay in elective surgery until the pneumonia has cleared, it would have been more prudent to operate electively rather than emergently upon someone with pneumobilia from common duct stones. The elective gall bladder and common duct surgery should have been performed as soon as her condition was stable, because elective gall bladder and common duct surgery have a much lower morbidity and mortality by about $30 \%$ than when the patient needs the surgery on an emergency basis [4].

\section{Conflict of interest None.}

\section{References}

1. Sherman SC, Tran H (2006) Pneumobilia: benign or life-threatening. J Emerg Med 30:147-153

2. Lee NK, Kim S, Lee JW, Lee SH, Kang DH, Kim DU, Kim GH, Seo HI (2010) MR appearance of normal and abnormal bile: correlation with imaging and endoscopic finding. Eur J Radiol 76:211-221

3. Lin YF, Chao HM, His SC (2009) Emphysematous cholecystitis with pneumobilia. Dig Surg 26:20-21

4. Roslyn JJ, Binns GS, Hughes EF, Saunders-Kirkwood K, Zinner MJ, Cates JA (1993) Open cholecystectomy. A contemporary analysis of 42, 474 patients. Ann Surg 218:129-137 